\title{
Photosensitivity and Acute Liver Insufficiency in Late- Onset Erythropoietic Protoporphyria with a Chromosome 18q Abnormality
}

\author{
Yuka Oshikawaa, e Satoshi Fukushima ${ }^{\mathrm{e}}$ Taiga Miyake $^{\mathrm{a}, \mathrm{e}}$ \\ Takeshi Kawaguchi $^{\text {b }}$ Kenta Motomurab \\ Yasuhiro Nakashima ${ }^{c}$ Kenichi Nakamura ${ }^{d}$ \\ Masatoshi Jinnin ${ }^{\mathrm{e}}$ Hironobu $\mathrm{Ihn}^{\mathrm{e}}$ \\ ${ }^{a}$ Department of Dermatology, ${ }^{b}$ Liver Unit, and Departments of ${ }^{c}$ Hematology \\ and ${ }^{\mathrm{d}}$ General Medicine, lizuka Hospital, Fukuoka, and ${ }^{\mathrm{e}}$ Department of \\ Dermatology and Plastic Surgery, Faculty of Life Sciences, Kumamoto \\ University, Kumamoto, Japan
}

\section{Key Words}

Liver damage $\cdot$ Hematological abnormality · Plasmapheresis · Red blood cell exchange

\begin{abstract}
Late-onset erythropoietic protoporphyria (EPP) is rare, and it is usually associated with an acquired somatic mutation of the ferrochelatase gene secondary to hematological malignancy such as myelodysplastic syndrome or myeloproliferative disorder. In $0.5-1 \%$ of patients with EPP, deposition of protoporphyrin in the liver leads to progressive liver insufficiency. Herein, we report the case of a 67-year-old female who developed EPP with typical photosensitivity and hemolytic anemia. Six months later, she was admitted with acute liver damage with a rapidly progressing course, and developed liver insufficiency. She recovered from the liver insufficiency after undergoing plasmapheresis and red blood cell exchange transfusion. A bone marrow examination revealed normal features; however, a cytogenetic analysis identified an abnormal clone of cells with a translocation between chromosomes $13 q 12$ and 18q21.1. This is the first report of a patient who recovered from liver insufficiency. The results of this report suggest that plasmapheresis and red blood cell exchange transfusion are effective for treating liver insufficiency in patients with late-onset EPP.
\end{abstract}




\section{Introduction}

Erythropoietic protoporphyria (EPP) is an inherited disorder of heme biosynthesis caused by ferrochelatase (FECH) deficiency. Patients normally present with the disease in early childhood. Onset in adulthood has rarely been reported, and is usually associated with an acquired somatic mutation of the FECH gene secondary to hematological malignancies such as myelodysplastic syndrome (MDS) or myeloproliferative disorder (MPD). In 0.5-1\% of patients with EPP, deposition of protoporphyrin in the liver leads to progressive liver insufficiency. Only two cases of liver disease in patients with late-onset EPP have been reported [1, 2], and both patients died due to liver insufficiency. Herein, we describe the case of a patient with a chromosome 18q abnormality who developed photosensitivity and acute liver insufficiency due to late-onset EPP. Our patient recovered after undergoing plasmapheresis and red blood cell (RBC) exchange transfusion.

\section{Case Report}

A 67-year-old female presented with erythema and pigmentation accompanied by swelling and slight sclerosis, predominantly over the face, forearms and hands that had been present for 2 months (fig. 1). No blisters or scars were noted. The patient complained of a severe burning sensation in sunexposed areas. Besides the presence of fatty liver determined during checkup, the patient was otherwise well and on no regular medications. Histology of a skin biopsy taken from the dorsal surface of the left hand showed extensive proliferation of a hyaline-like material positive for periodic acid-Schiff stain in and around superficial dermal blood vessels. Laboratory tests showed an erythrocyte protoporphyrin level of $919.6 \mu \mathrm{g} / \mathrm{dl}$ (normal range (NR): $91.1 \pm 14.9$ ) and a feces protoporphyrin level of $118.13 \mu \mathrm{g} / \mathrm{dl}$ (NR: $1.73 \pm 1.00$ ), primarily free form, not complexed with zinc, suggesting a diagnosis of EPP. The patient had anemia (hemoglobin: $7.7 \mathrm{~g} / \mathrm{dl}$ ), was normocytic and her haptoglobin level was decreased (1.0 mg/dl (NR: 19-170 mg/dl)). Therefore, the diagnosis was considered to be hemolytic anemia. Liver function tests were normal. A bone marrow examination revealed normal features. A cytogenetic analysis of the bone marrow cells identified an abnormal clone of cells with a translocation between chromosomes 13q12 and 18q21.1 (fig. 2 ).

Six months later, the patient presented at the emergency room with a complaint of lower abdominal pain. Biochemical tests of liver function showed abnormal counts. Although the patient was treated with photo-protection, a large amount of fluid infusion and oral cimetidine, cholestyramine, ursodeoxycholic acid, nicotinate and lactulose, her condition worsened. We attempted plasmapheresis (exchanged 7 liters of plasma) and RBC exchange infusion (we removed $400 \mathrm{ml}$ of whole blood and transfused an equivalent amount of red cell concentrates) for 3 days, and the patient's liver function improved remarkably (fig. 3 ). At present, the patient receives blood transfusions periodically for anemia in an outpatient clinic.

\section{Discussion}

EPP is an inherited disorder of the heme metabolic pathway characterized by accumulation of protoporphyrin in blood, erythrocytes and tissues, and cutaneous manifestations of photosensitivity. In most patients, EPP results from a mutation of the gene that encodes FECH in the long arm of chromosome 18. This enzyme catalyzes the insertion of ferrous iron into the protoporphyrin IX ring to form heme [3]. Patients normally present with the disease in early childhood. Adult onset is rare, and to our knowledge, only eleven such cases have been reported. Eight of these cases were associated with hematological malignancies such as MDS (often the sideroblastic 
anemia subtype) or MPD [1, 2, 4-9]. Five cases were associated with chromosome 18 deficiency [1, 6-9], and two with liver dysfunction [1, 2]. Late-onset EPP has been described previously in association with hematological malignancies, where it is usually caused by proliferation of a clone of hematopoietic cells in which one FECH allele has been deleted by a somatic mutation. An abnormal clone of cells with a translocation between chromosomes 13q12 and 18q21.1 was identified in the bone marrow cells of our patient. We speculate that this chromosomal translocation, including chromosome 18q with the breakpoint in the large 18q21 region in which the FECH gene lies at 18q21.31, caused EPP. No point mutations in the FECH gene were identified in germ-line DNA from peripheral blood cells. This indicates that the somatic mutation in our patient's hematopoietic cells was not an inherited, previously clinically latent FECH mutation.

As protoporphyrin is a lipophilic molecule that is excreted by the liver, EPP patients are at risk of developing cholelithiasis with obstructive episodes and chronic liver disease that rapidly evolve to acute liver insufficiency. Cholelithiasis and mild liver disease frequently occur in patients with EPP (10-20\%), while only $5 \%$ of patients with liver damage presents with an acute liver insufficiency. Progressive hepatocellular disease ultimately leads to cholestatic hepatocellular failure, which often has an acute onset, and a rapidly progressive, irreversible course [10]. When unexplained jaundice occurs in a patient with EPP, the hepatic disease is generally so advanced that the patient dies within a few months (range: 1 month to 2 years, median: 3.5 months) unless liver transplantation is performed. There are no specific factors that influence the development of hepatocellular disease. Previous studies have revealed that increases in coproporphyrin urinary excretion, changes in isomer predominance from isomer III to isomer I, and increases in the levels of protoporphyrinemia may precede this complication [10]. In our patient, the level of urinary porphyrin was elevated before the liver dysfunction appeared. She was treated with plasmapheresis and RBC exchange transfusion, which resulted in the resolution of the liver insufficiency. Previously, only two cases of patients with liver disease in late-onset EPP have been reported and both patients died due to liver insufficiency [1,2]. These patients did not receive plasmapheresis or RBC exchange transfusion. Plasmapheresis has been previously reported to be an adjunctive therapy for patients with advanced hepatic EPP. The use of plasmapheresis has been limited to a few case reports of patients who underwent liver transplantation. Circulating levels of protoporphyrin can be decreased by plasmapheresis and RBC exchange transfusion. Because the concentration of protoporphyrins is several-fold higher inside the RBC than in plasma, RBC exchange therapy might absorb excess protoporphyrins from the plasma and serve as an effective therapy to reduce protoporphyrin load in patients with advanced hepatic EPP $[11,12]$. This is the first report of a patient who recovered from liver insufficiency, and the results of this report suggest that plasma exchange and RBC exchange transfusion are effective in treating liver insufficiency in patients with late-onset EPP. The bone marrow of our patient showed an abnormality in chromosome 18, similar to that observed in other patients with late-onset EPP with MDS or MPD. Although our patient's bone marrow was normocellular on admission, chromosome abnormalities and clonally proliferation were later observed. Careful follow-up is needed to monitor the occurrence of MDS or MPD. Furthermore, management of this uncommon complication of hematological malignancies such as MDS or MPD should include measures to prevent the development of fatal protoporphyric liver disease. 


\section{Disclosure Statement}

The authors have no conflicts of interest to disclose.


Fig. 1. Patient's face (a) and forearms and hands (b) showing swelling and slight sclerosis accompanied by erythema and pigmentation. 


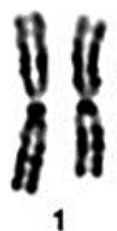

1
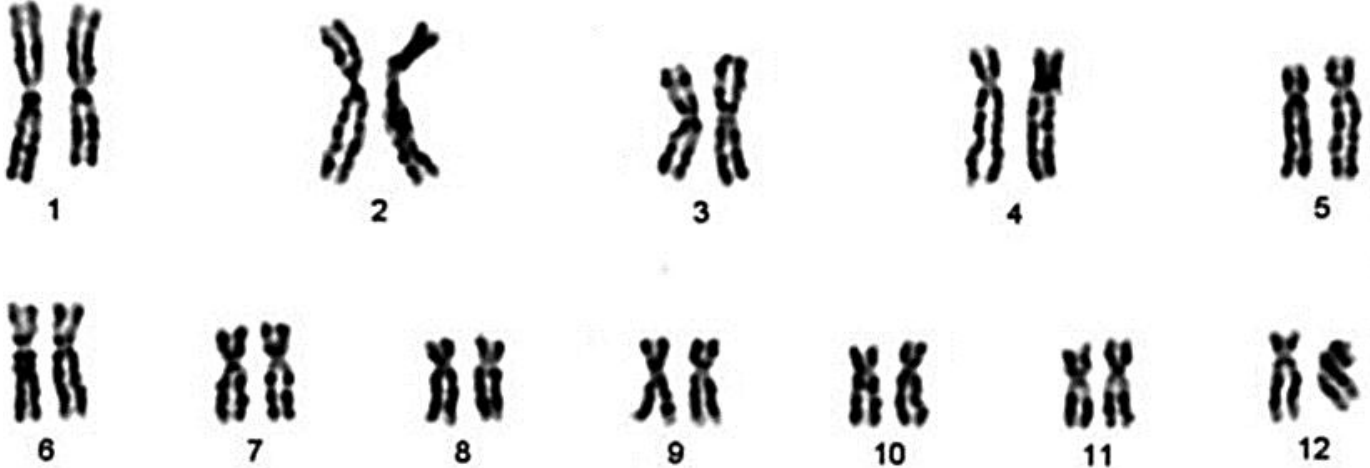

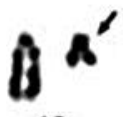

13
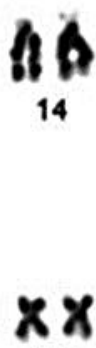

20
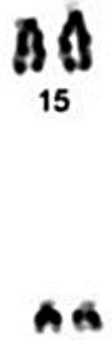

21

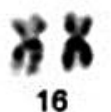

$\cot _{17}^{\text {से }}$

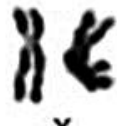

22
12

Fig. 2. Bone marrow cytogenetic analysis revealed a translocation between chromosomes $13 q 12$ and 18q21.1.

$\rightarrow$ AST $\rightarrow$ ALT $\rightarrow-H_{b} \rightarrow-T-B i l$

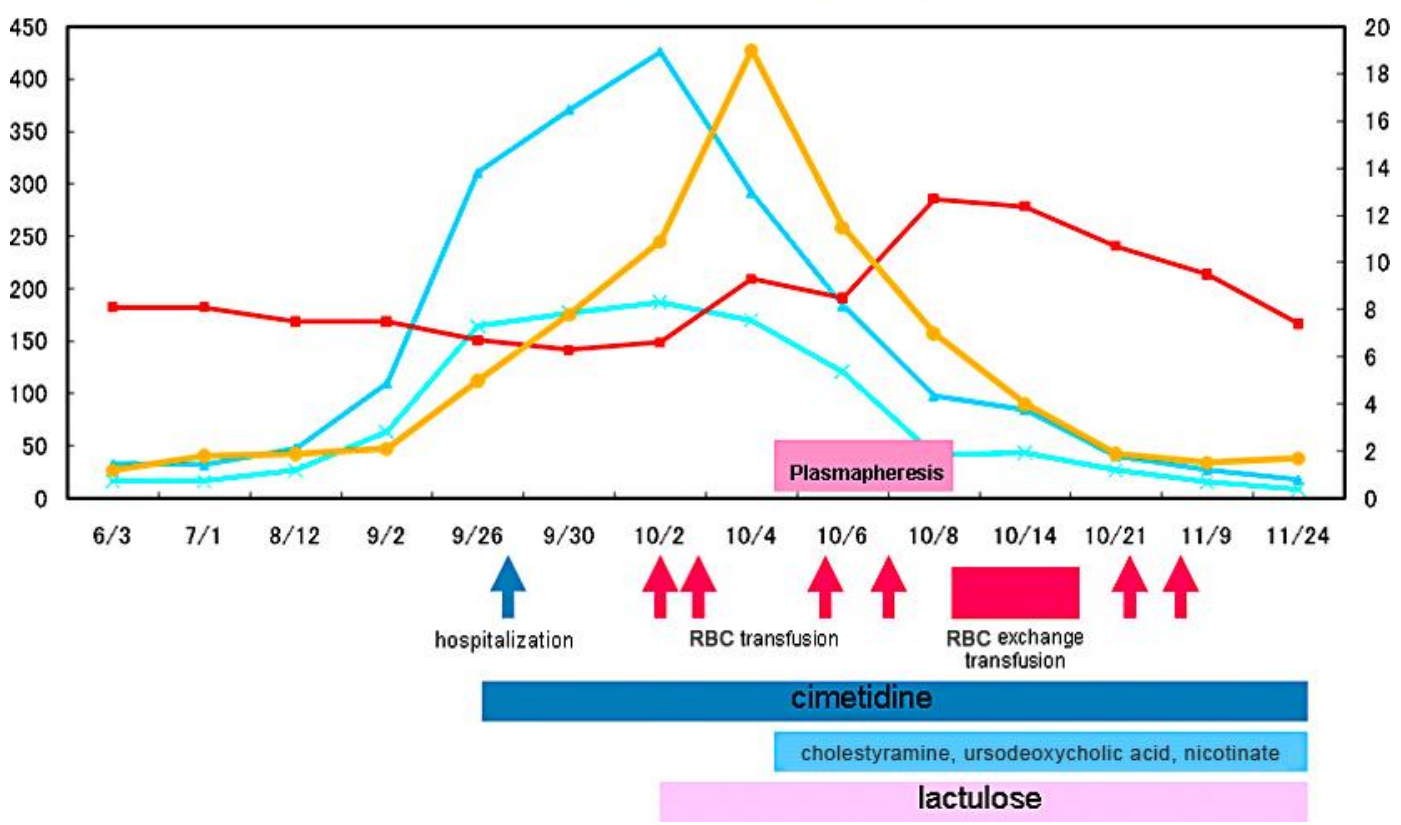

Fig. 3. Clinical course of the patient. AST = Aspartate transaminase; ALT = alanine transaminase; $\mathrm{Hb}=$ hemoglobin; T-Bil = total-bilirubin. 


\section{References}

1 Goodwin RJ, Kell WJ, Laidler P, Long CC, Whatley SD, Mckinley M, Badminton MN, Burnett AK, Williams GT, Elder GH: Photosensitivity and acute liver injury in myeloproliferative disorder secondary to lateonset protoporphyria caused by deletion of a ferrochelatase gene in hematopoietic cells. Blood 2006;107:60-62.

-2 Shirota T, Yamamoto H, Hayashi S, Fujimoto H, Harada Y, Hayashi T: Myelodysplastic syndrome terminating in erythropoietic protoporphyria after 15 years of aplastic anemia. Int J Hematol 2000;72:44-47.

-3 Lecha M, Puy H, Deybach JC: Erythropoietic protoporphyria. Orphanet J Rare Dis 2009;4:19.

-4 Berroeta L, Man I, Goudie DR, Whatley SD, Elder GH, Ibbotson SH: Late presentation of erythropoietic protoporphyria: case report and genetic analysis of family members. Br J Dermatol 2007;157:10301031.

-5 Sato Y, Motoji T, Yamada O, Ito Y, Katahira J, Takahashi M, Mazatani R, Mizoguchi H, Okada M: A case of sideroblastic anemia with dermal photosensitivity and increased erythrocyte protoporphyrin (in Japanese). Rinsho Ketsueki 1981;22:1971-1976.

-6 Sarkany RP, Ross G, Willis F: Acquired erythropoietic protoporphyria as a result of myelodysplasia causing loss of chromosome 18. Br J Dermatol 2006;155:464-466.

7 Lim HW, Cooper D, Sassa S, Dosik H, Buchness MR, Soter NA: Photosensitivity, abnormal porphyrin profile, and sideroblastic anemia. J Am Acad Dermatol 1992;27:287-292.

8 Bharati A, Badminton MN, Whatley SD, O’Brien DV, Bell HK: Late-onset erythropoietic protoporphyria in association with haematological malignancy. Clin Exp Dermatol 2006;31:668-670.

-9 Rothstein G, Lee R, Cartwright GE: Sideroblastic anemia with dermal photosensitivity and greatly increased erythrocyte protoporphyrin. N Engl J Med 1969;280:587-590.

10 Todd DJ: Erythropoietic protoporphyria. Br J Dermatol 1994;131:751-766.

11 Dellon ES, Szczepiorkowski ZM, Dzik WH, Graeme-Cook F, Bloomer JR, Cosimi AB, Chung RT: Treatment of recurrent allograft dysfunction with intravenous hematin after liver transplantation for erythropoietic protoporphyria. Transplantation 2002;73:911-915.

12 Eichbaum QG, Dzik WH, Chung RT, Szczepiorkowski ZM: Red blood cell exchange transfusion in two patients with advanced erythropoietic protoporphyria. Transfusion 2005;45:208-213. 